\title{
Mulliken Occupation as the Indicator of Transition to Superconducting State in $\mathrm{SrFe}_{2} \mathrm{As}_{2}$ and $\mathrm{BaFe}_{2} \mathrm{As}_{2}$
}

\author{
J. Goraus* \\ Institute of Physics, University of Silesia, Uniwersytecka 4, PL-40-007 Katowice, Poland
}

\begin{abstract}
Basing on the $a b$ initio calculations performed within full potential local orbital minimum basis method, the Mulliken occupation of the $\mathrm{Sr} 5 s\left(\mathrm{n}_{5 s}\right)$ and Ba $6 p\left(\mathrm{n}_{6 p}\right)$ states can serve as an indicator of transition to the superconducting state in doped $\mathrm{SrFe}_{2} \mathrm{As}_{2}$ and $\mathrm{BaFe}_{2} \mathrm{As}_{2}$ compounds where the iron was substituted with cobalt or in the pristine compounds under pressure. The estimated pressure, at which both compounds exhibit superconductivity based on $\mathrm{Sr} 5 s$ and Ba $6 p$ occupation is in good agreement with the recently published experimental data.
\end{abstract}

DOI: 10.12693/APhysPolA.130.655

PACS/topics: 74.40.Kb, 71.15.Mb, 74.25.Dw

\section{Introduction}

The iron-based superconductors have been recently very intensively studied due to the relatively high temperature at which they can still exist in the superconducting state and similarities to the well known copper oxide high- $\mathrm{T}_{c}$ superconductors [1]. The first reports concerned $R \mathrm{FeAsO}$ ( $\mathrm{R}=$ rare earth element) type compounds, with quasi-two-dimensional tetragonal structure where it was postulated that electron count within charged $(\mathrm{LaO})^{\delta+}$ and $(\mathrm{FeAs})^{\delta-}$ layers plays an important role in suppressing the antiferromagnetic order and emergence of the superconducting state [2]. Later other superconducting systems with $(\mathrm{FeAs})^{\delta-}$ layers were discovered, which suggested that these layers are crucial for superconducting state formation. These compounds crystallize in $\mathrm{ThCr}_{2} \mathrm{Si}_{2}$ crystal structure with alternating layers of alkaline earth or rare earth metals separated by the $(\mathrm{FeAs})^{\delta-}$ layers.

$\mathrm{SrFe}_{2} \mathrm{As}_{2}$ and $\mathrm{BaFe}_{2} \mathrm{As}_{2}$ belong to this class, moreover $\mathrm{BaFe}_{2} \mathrm{As}_{2}$ gained considerable interest when it was reported that it exhibits moderately high critical temperature of $T \approx 38 \mathrm{~K}$, when barium sites are doped with potassium [3]. Both systems are tetragonal $(I 4 / \mathrm{mmm}$ space group) at the ambient conditions, and both undergo a structural transition to the orthorombic phase (Fmmm space group) at low temperatures (203 K for $\mathrm{SrFe}_{2} \mathrm{As}_{2}$ [4] and $140 \mathrm{~K}$ for $\mathrm{BaFe}_{2} \mathrm{As}_{2}$ [3]). For both systems the superconducting phase is orthorombic and the superconductivity can be induced by either application of external pressure or by doping.

For $\mathrm{SrFe}_{2} \mathrm{As}_{2}$ the superconductivity was reported upon substitution of $20-40 \%$ of Fe by Co [5] or by applying pressure above $2 \mathrm{GPa}[6]$ or $3 \mathrm{GPa}[7,8]$. In tetragonal phase at the room temperature the bulk modulus of $\mathrm{SrFe}_{2} \mathrm{As}_{2}$ is about $\approx 46 \mathrm{GPa}[9]$, however, upon applying

*e-mail: jerzy.goraus@us.edu.pl a pressure above $10.7 \mathrm{GPa} \mathrm{SrFe}_{2} \mathrm{As}_{2}$ undergoes a transition to collapsed tetragonal phase with much higher bulk modulus $\approx 112 \mathrm{GPa}$.

In a similar manner $\mathrm{BaFe}_{2} \mathrm{As}_{2}$ exhibits superconductivity at pressures higher than $0.8-1.5 \mathrm{GPa}$ [10] or when $2.5-18 \%$ of Fe atoms are substituted by Co atoms [11, 12]. At the ambient conditions within tetragonal phase the bulk modulus of pristine $\mathrm{BaFe}_{2} \mathrm{As}_{2}$ is about $59 \mathrm{GPa}$ [13], whereas at the high pressures 26-29 GPa structural phase transition to collapsed tetragonal phase takes place [14]. The transition from the metallic to superconducting state caused by doping or external pressure, depends on the electron concentration and charge transfer between $(\mathrm{FeAs})^{\delta-}$ and $\mathrm{Sr} / \mathrm{Ba}$ layers [3]. One can expect therefore that charge transfer to the $\mathrm{Sr} / \mathrm{Ba}$ sites should indicate the onset of superconductivity.

The calculations performed for $\mathrm{SrFe}_{2} \mathrm{As}_{2}$ and $\mathrm{BaFe}_{2} \mathrm{As}_{2}$ doped with $\mathrm{Co}$ or under pressure indicate that the Mulliken occupation [15] of Sr-5s and Ba-6 $p$ states indeed reflects the superconducting state formation. The $6 p$ states which are unoccupied in a free $\mathrm{Ba}$ atom do exhibit some finite occupation in a $\mathrm{BaFe}_{2} \mathrm{As}_{2}$ crystal, which also reflects the charge transfer from the $(\mathrm{FeAs})^{\delta-}$ layers. We have shown that transition from the Kondo insulator to metallic state depends on critical $5 d$ electron occupation of rare earth metal [16, 17], here for the transition to superconducting state Sr-5s and Ba-6p occupation seems to play a similar role.

\section{Calculation details}

Band structure calculations were performed within minimum basis full potential local orbital (FPLO) method (FPLO 5.00-20 computer code) [18] with the local spin density approximation (LSDA) exchangecorrelation potential in the form proposed by Perdew and Wang [19]. The basis was chosen as follows: as a valence states were considered $(6 s, 6 p, 5 d)$ for $\mathrm{Ba},(5 s, 5 p, 4 d)$ for $\mathrm{Sr}$ and $(4 s, 4 p, 3 d)$ for $\mathrm{Fe}$, Co, As; as the semicore states were considered $(5 s, 5 p)$ for $\mathrm{Ba},(4 s, 4 p)$ for $\mathrm{Sr}$ and $(3 s, 3 p)$ for Fe, Co, As. Structural data was taken from [5] for 
$\mathrm{SrFe}_{2} \mathrm{As}_{2}$ and from [2] for $\mathrm{BaFe}_{2} \mathrm{As}_{2}$. Calculations were performed in scalar relativistic, spin-resolved way.

The effect of pressure was studied by performing calculations for the unit cell uniformly scaled down to $85 \%$ of the experimental volume. Pressure was determined from the formula $p=B \ln V_{0} / V$ where $B$ stands for bulk modulus, $V_{0}$ for the equilibrium volume and $V$ for the scaled unit cell volume. The experimental unit cell volume was taken as $V_{0}$, whereas bulk modulus was taken from Ref. [9] for $\mathrm{SrFe}_{2} \mathrm{As}_{2}$ and Ref. [13] for $\mathrm{BaFe}_{2} \mathrm{As}_{2}$.

The substitution of $\mathrm{Fe}$ atoms with $\mathrm{Co}$ was investigated within virtual crystal approximation which is justified by the fact that these two elements are neighbours in periodic table. Calculations were performed for $280 \mathrm{k}$ points in the irreducible wedge of the Brillouin zone and their convergence was carefully checked.

Population of particular states was calculated using the Mulliken charge analysis [15]. Such analysis have its well known problems, i.e. it can give negative occupations or occupations above the maximum number for a given orbital, moreover it is also a rather approximate tool to study crystal. It should be emphasised that in this report the main purpose was the study of changes of the particular occupations with pressure or upon doping, and not the absolute occupation number which has indeed little meaning. The changes of the charge symmetry coming from the changes of the particular orbital occupations are here the most important point, and as the presented results show rather small occupation changes of the order $10^{-4}$ electron $(e)$. All presented results were carefully checked to have charge convergence better than $1 \times 10^{-5} e$ per unit cell.

\section{Results}

Figure 1 shows the Mulliken Sr $5 s$ occupation (upper part) and the Mulliken Sr $5 p$ occupation (lower part) calculated for the $\mathrm{Sr}\left(\mathrm{Fe}_{1-x} \mathrm{Co}_{x}\right)_{2} \mathrm{As}_{2}$. For this series it was reported from resistivity and susceptibility measurements that superconductivity occurs for Co concentrations $0.2<x<0.4$ [5], which is marked in Fig. 1 as a gray shaded area. Full circles denote the Mulliken occupations for experimental lattice parameters and As $z$ position, whereas blue crosses represent occupations for experimental lattice parameters with As atoms shifted by $0.5 \%$ in $z$ direction. Red stars correspond to experimental As position, but lattice parameters scaled down by $1.5 \%$. The shape of the curves is essentially the same, therefore one can conclude that it is possible to chose a critical (or threshold) value of $\mathrm{Sr} 5 s$ population, which separates the superconducting state from the normal state. The absolute number for this critical occupation would be different for these three structural models considered, but the concentrations range below a threshold would correspond to $0.2<x<0.35$ in all those cases assuming that the threshold value is chosen at $x=0.2$. This concentration range is in good agreement with the region where the superconductivity was reported from experimental data. For experimental lattice parameters and experimental As position the threshold value is $n_{5 s}<0.204403$ electrons $(e)$ which is marked by a dashed line in Fig. 1. In Fig. 2 the $\mathrm{Sr} 5 s$ occupation calculated vs. external pressure for the pristine $\mathrm{SrFe}_{2} \mathrm{As}_{2}$ compound is shown, here again the same critical value $n_{5 s}=0.204403 e$ is denoted by a dashed line. The shaded region corresponds to $n_{5 s}<0.204403 e$, where the superconductivity should occur according to the $\mathrm{Sr} 5 s$ occupation criterion suggested above for the $\mathrm{Sr}\left(\mathrm{Fe}_{1-x} \mathrm{Co}_{x}\right)_{2} \mathrm{As}_{2}$ series. We see that the threshold value is reached for pressures $p>2.95 \mathrm{GPa}$, where it was indeed reported that the superconducting state is formed in $\mathrm{SrFe}_{2} \mathrm{As}_{2}[7,8]$.

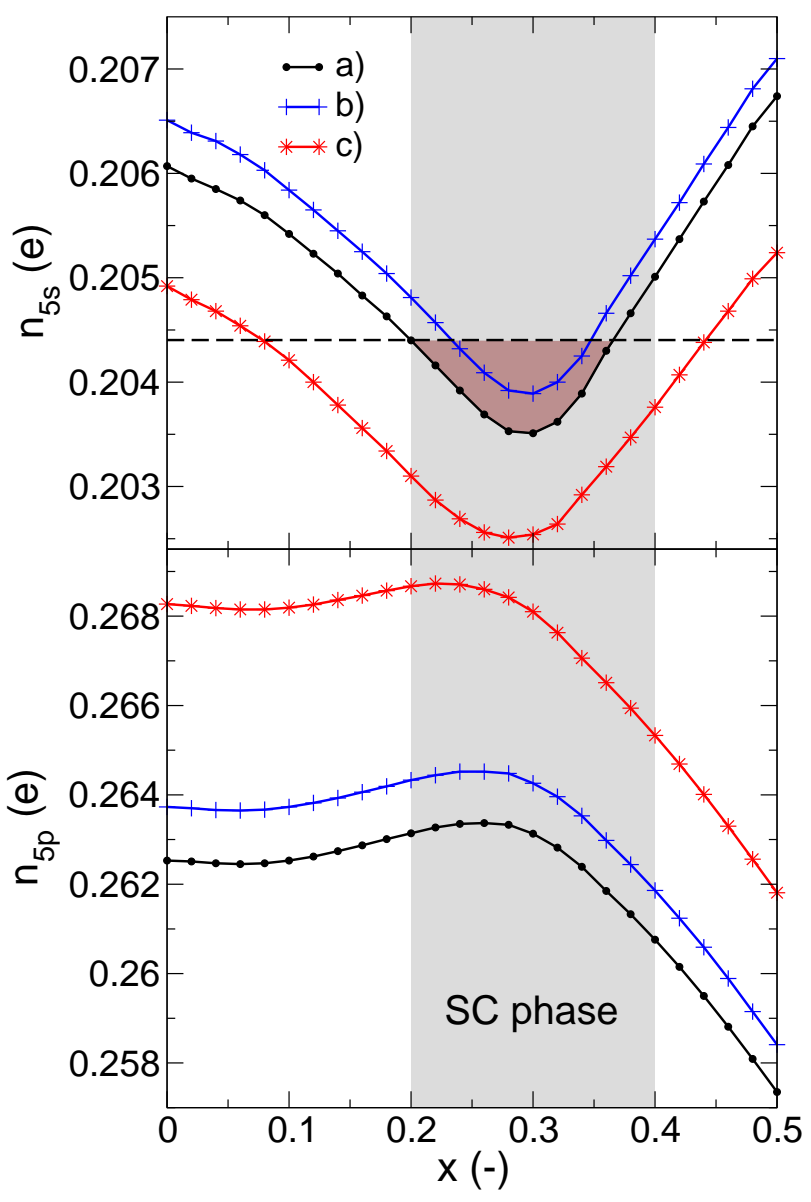

Fig. 1. Mulliken occupation of $\mathrm{Sr} 5 s$ (upper part) and Sr $5 p$ states (lower part) for the $\mathrm{Sr}\left(\mathrm{Fe}_{1-x} \mathrm{Co}_{x}\right)_{2} \mathrm{As}_{2}$ series. Gray shading denotes region where superconductivity was reported from experimental data [5]. The calculations were performed for unit cells with: (a) experimental lattice parameters and experimental As $8 i$ position, (b) experimental lattice parameters and As shifted by $0.5 \%$ in $z$ direction from the origin, and (c) lattice parameters uniformly scaled down by $1.5 \%$ and experimental As $8 i$ position. Dashed line placed at $n_{5 s}=0.204403$ electrons serves as a guidance for eye and indicates critical $5 s$ occupation below which a superconducting state is formed. 


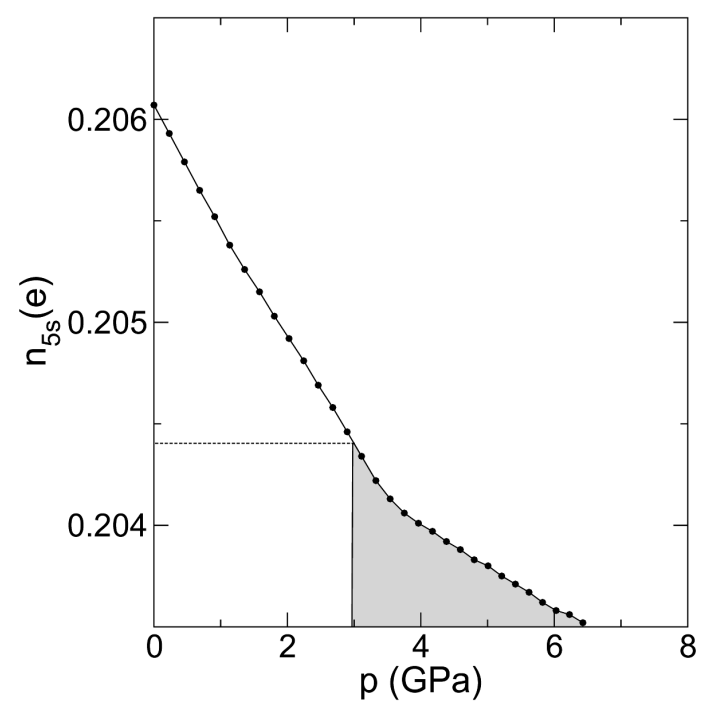

Fig. 2. Mulliken occupation of $\mathrm{Sr} 5 s$ states vs. pressure calculated for the pristine $\mathrm{SrFe}_{2} \mathrm{As}_{2}$ compound. The same value of critical occupation $\mathrm{n}_{5 s}=0.204403 e$ is denoted by the horizontal line, which corresponds to critical pressure at which superconducting state is formed $\approx 2.95 \mathrm{GPa}$.

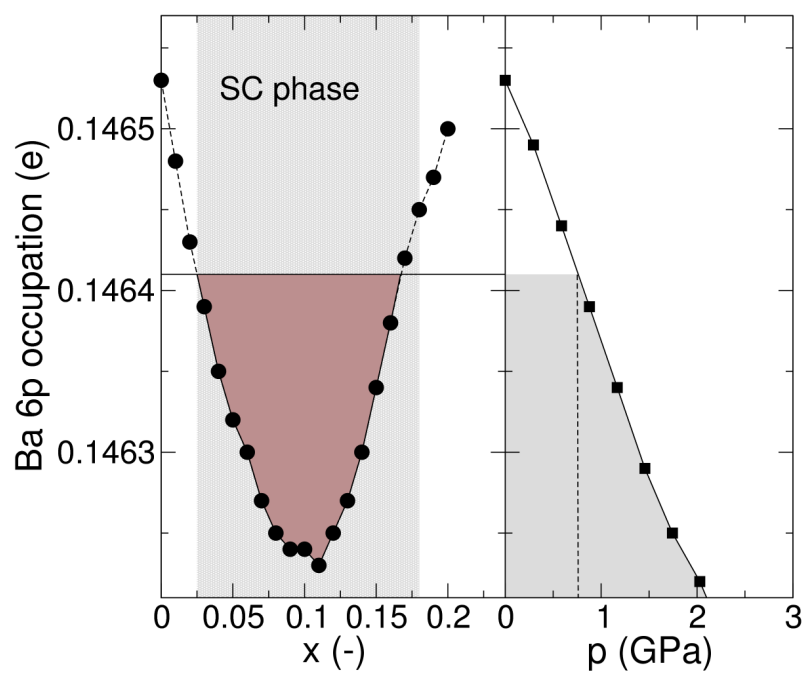

Fig. 3. Left part presents Mulliken occupation of $\mathrm{Ba} 6 p$ states vs. Co concentration $x$ for the $\mathrm{Ba}\left(\mathrm{Fe}_{1-x} \mathrm{Co}_{x}\right)_{2} \mathrm{As}_{2}$ series. Shaded area (SC) represents superconducting region known from experimental data $0.025<x<0.18$. Horizontal line denotes threshold value $n_{6 p}<0.14641 e$ which coincides with the superconducting region. Right part shows Mulliken occupation of $\mathrm{Ba} 6 p$ states vs. pressure for the pristine $\mathrm{BaFe}_{2} \mathrm{As}_{2}$ compound, where the same threshold value $n_{6 p}<0.14641 e$ is denoted by gray shaded area. Basing on the $\mathrm{Ba} 6 p$ occupation superconductivity should appear at pressure above $0.8 \mathrm{GPa}$.

Similar calculations performed for the $\mathrm{Ba}\left(\mathrm{Fe}_{1-x} \mathrm{Co}_{x}\right)_{2} \mathrm{As}_{2}$ series show that the $\mathrm{Ba} 6 p$ occupation also exhibits non-monotonic dependence vs. Co concentration $x$ (Ba $6 p$ states are not occupied in a free $\mathrm{Ba}$ atom but do exhibit some finite occupation in a crystal). Figure 3 presents the calculated
Mulliken occupation $n_{6 p}$ of the $\mathrm{Ba} 6 p$ states for the series $\mathrm{Ba}\left(\mathrm{Fe}_{1-x} \mathrm{Co}_{x}\right)_{2} \mathrm{As}_{2}$ vs. Co concentration $x$ (left part), and the calculated Mulliken occupation $n_{6 p}$ vs. external pressure for the pristine $\mathrm{BaFe}_{2} \mathrm{As}_{2}$ compound. The critical occupation $n_{6 p}<0.14641$ below which a superconducting state is formed can be related to concentration range $0.025<x<0.18$ or pressures above $0.8 \mathrm{GPa}$. We see that again this numbers agree well with the experimental reports where superconductivity was postulated for the $0.025<x<0.2$ concentration range $[11,12]$ or for the pressure above $0.8-1.5 \mathrm{GPa}[10]$.

For both investigated series although the Co doping increases the valence electron count, it at first reduces the $n_{5 s}$ or $n_{6 p}$ occupation and increases the number of holes. In this way hole doping is accomplished in similar way like in $\mathrm{Ba}_{0.6} \mathrm{~K}_{0.4} \mathrm{Fe}_{2} \mathrm{As}_{2}$ where substitution of $\mathrm{Ba}$ with $\mathrm{K}$ takes $0.15-2$ electrons from the FeAs unit [20]. The presented values of the critical occupations serve here only for comparison of the changes with respect to doping or under pressure, and their absolute values are here less important. Within the $\mathrm{Sr}\left(\mathrm{Fe}_{1-x} \mathrm{Co}_{x}\right)_{2} \mathrm{As}_{2}$ and $\mathrm{Ba}\left(\mathrm{Fe}_{1-x} \mathrm{Co}_{x}\right)_{2} \mathrm{As}_{2}$ series only the $\mathrm{Sr} 5 s$, Sr $5 p$ and $\mathrm{Ba}$ $6 p$ were found to exhibit non-monotonic behaviour with respect to the concentration of dopant.

\section{Conclusions}

It has been shown that the Mulliken occupations of $\mathrm{Sr}$ $5 s$ states for $\mathrm{SrFe}_{2} \mathrm{As}_{2}$ and $\mathrm{Ba} 6 p$ states for $\mathrm{BaFe}_{2} \mathrm{As}_{2}$ can serve as indicator of the superconducting transition, however, in contrast to the transition from the Kondo insulating to metallic state $[16,17]$ there is no sign of abrupt change of the occupation with respect to doping or under pressure at the border between the superconducting and the non-superconducting state. Moreover, the magnitude of occupation changes is also much smaller. The transition to superconducting state is therefore much more subtle and the charge reorganization exhibits gradual changes across the control parameter (pressure or doping) for the $\mathrm{SrFe}_{2} \mathrm{As}_{2}$ and $\mathrm{BaFe}_{2} \mathrm{As}_{2}$ compounds. The charge transfer to the $(\mathrm{FeAs})^{\delta-}$ layers which leads to superconductivity has therefore different symmetry for the two investigated compounds, for both cases, however, hole doping within $5 s$ or $6 p$ shell is accomplished. The estimated pressures at which both systems undergo a transition to superconducting state agrees well with the reported experimental data for the experimental structural parameters. The non-monotonic behaviour of $\mathrm{Sr} 5 s$ or Ba $6 p$ occupation vs. doping is, however, not particularly sensitive to the lattice parameters or As position.

\section{References}

[1] A.D. Christianson, E.A. Goremychkin, R. Osborn, S. Rosenkranz, M.D. Lumsden, C.D. Malliakas, I.S. Todorov, H. Claus, D.Y. Chung, M.G. Kanatzidis, R.I. Bewley, T. Guidi, Nature 456, 930 (2008).

[2] M. Rotter, M. Tegel, D. Johrendt, Phys. Rev. Lett. 101, 107006 (2008). 
[3] E. Aktürk, S. Ciraci, Phys. Rev. B 79, 184523 (2009).

[4] M. Tegel, M. Rotter, V. Weiß, F.M. Schappacher, R. Pöttgen, D. Johrendt, J. Phys. Condens. Matter 20, 452201 (2008).

[5] A. Leithe-Jasper, W. Schnelle, C. Geibel, H. Rosner, Phys. Rev. Lett. 101, 207004 (2008).

[6] H. Takahashi, H. Okada, K. Igawa, K. Arii, Y. Kamiharai, S. Matsuishi, M. Hirano, H. Hosono, K. Matsubayashi, Y. Uwatoko, J. Phys. Soc. Jpn. $\mathbf{7 7}$ Suppl. C, 78 (2008).

[7] P.L. Alireza, Y.T.C. Ko, J. Gillett, C.M. Petrone, J.M. Cole, G.G. Lonzarich, S.E. Sebastian, J. Phys. Condens. Matter 21, 012208 (2009).

[8] M. Kumar, M. Nicklas, A. Jesche, N. Caroca-Canales, M. Schmitt, M. Hanfland, D. Kasinathan, U. Schwarz, H. Rosner, C. Geibel, Phys. Rev. B 78, 184516 (2008).

[9] W.O. Uhoya, J.M. Montgomery, G.M. Tsoi, Y.K. Vohra, M.A. McGuire, A.S. Sefat, B.C. Sales, S.T. Weir, J. Phys. Condens. Matter 23, 122201 (2011).

[10] A. Mani, N. Ghosh, S. Paulraj, A. Bharathi, C.S. Sundar, Europhys. Lett. 87, 17004 (2009).

[11] J.-H. Chu, J.G. Analytis, C. Kucharczyk, I.R. Fisher, Phys. Rev. B 79, 014506 (2009).

[12] A. Lucarelli, A. Dusza, F. Pfuner, P. Lerch, J.G. Analytis, J.-H. Chu, I.R. Fisher, L. Degiorgi, New J. Phys. 12, 073036 (2010).
[13] J.-E. Jorgensen, T.C. Hansen, Eur. Phys. J. B 78, 411 (2010).

[14] R. Mittal, S.K. Mishra, S.L. Chaplot, S.V. Ovsyannikov, E. Greenberg, D.M. Trots, L. Dubrovinsky, Y. Su, Th. Brueckel, S. Matsuishi, H. Hosono, G. Garbarino, arXiv:1007.2320.

[15] R.S. Mulliken, J. Chem. Phys. 23, 1833 (1955).

[16] J. Goraus, A. Ślebarski, Phys. Lett. A 375, 3469 (2011).

[17] J. Goraus, A. Ślebarski, Solid State Commun. 151, 1318 (2011)

[18] K. Koepernik, H. Eschrig, Phys. Rev. B 59, 1743 (1999); I. Opahle, K. Koepernik, H. Eschrig, Phys. Rev. B 60, 14035 (1999); K. Koepernik, B. Velicky, R. Hayn, H. Eschrig, Phys. Rev. B 55, 5717 (1997); H. Eschrig, M. Richter, I. Opahle, in: Relativistic Electronic Structure Theory, Part 2, Applications, Ed. P. Schwerdtfeger, Theoretical and Computational Chemistry, Vol. 13, Elsevier, 2004, p. 723; H. Eschrig, K. Koepernik, I. Chaplygin, J. Solid State Chem. 176, 482 (2003); FPLO Homepage.

[19] J.P. Perdew, Y. Wang, Phys. Rev. B 45, 13244 (1992); D.M. Ceperley, B.J. Alder, Phys. Rev. Lett. 45, 566 (1980).

[20] M. Rotter, M. Tegel, D. Johrendt, Phys. Rev. Lett. 101, 107006 (2008). 